\title{
The Potential Adverse Reactions of Administering Combination Therapy in Covid-19 Patients
}

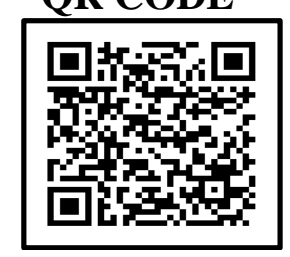

\section{VIKRANT KHULLAR}

A definite treatment modality for coronavirus disease 2019 (COVID-19) has still not come into picture. With the rise of COVID-19 pandemic, a few drugs have come into light as empirical treatment for this infection. This review focusses on existing approaches to the treat COVID-19 patients with antimalarial drugs and antibiotics analyzing the adverse reactions and interactions of concomitantly administering these drugs. We will also discuss the possibilities of alternate methods to treat this disease.

KEYWORDS: Coronavirus, COVID-19, Anti-malarial, Hydroxychloroquine, Azithromycin

\section{INTRODUCTION}

The deadly coronavirus disease 2019 is caused by the severe acute respiratory syndrome coronavirus 2 (SARS-CoV-2). COVID-19 has spread rapidly across the globe with countless confirmed cases deaths. The severity of COVID-19 is influenced by various factors such as age, gender, ethnicity, and comorbid conditions. Although many therapeutic treatments have been suggested, there is no approved antiviral treatment specific for COVID-19. Countless challenges associated with this pandemic has stressed the medical professionals to seek therapeutic strategies, including the use of well-researched drugs for new indications. Subjects with cardiovascular issues seem to be at an elevated risk of unfavorable outcomes in the COVID-19 infection.

The respiratory symptoms dominate in the clinical presentation of COVID-19, a few subjects may also have significant impairment of the cardiovascular system. In a published report on patients with COVID-19, 68\% had at least one concurrent health issue, including hypertension, diabetes mellitus and other cardiovascular diseases. ${ }^{1}$ There is a need to analyze and understand the safety of the therapeutic measures used in patients with COVID-19 due to the possible cardiac adverse effects of these drugs, keeping in mind that most of the infected subjects are aged and have more possibility of suffering from cardiovascular diseases. The progress in finding new therapeutic indications for the currently used drugs is advancing at a great pace and the active use of azithromycin with antimalarial drugs is being practiced globally. The main reason behind the use of this combination is rooted on the pathogenetic understanding of the possible mechanism of antiviral action of chloroquine and hydroxychloroquine. This is apparently based on blocking the penetration of virus into cells by inhibition of host cell glycosylation and endosomal acidification.

The analysis of established literature gives rise to apprehension about the broad use of this drug combination in COVID-19 patients. Chloroquine is an aminoquinoline derivative, initially used as an antiprotozoal drug. Its mechanism of action is associated with inhibition of nucleic acid synthesis in cells, and the drug has moderate immunosuppressive and anti-inflammatory effect. It is used for rheumatoid arthritis, systemic lupus erythematosus, autoimmune glomerulonephritis, sarcoidosis, and scleroderma. It also has an antiarrhythmic effect due to decreased excitability of the heart muscle. It has many contraindications like hypersensitivity, hepatic failure, renal failure, bone marrow depression, cardiac injury, rhythm disorder, neutropenia, psoriatic arthritis, porphyrinuria and pregnancy. The drug dosing restrictions are glucose-6-phosphate dehydrogenase deficiency, retinopathy, epilepsy, myasthenia, severe gastrointestinal disorders, and concomitant use of hepatotoxic agents. It also interacts with many drugs including cimetidine, penicillamine, phenylbutazone, cytostatics, levamisole, glucocorticoids, ethanol and cardiac glycosides. The gastrointestinal and skin 
manifestations of this drug are considered to be nonserious adverse events, and retinal, neuromuscular, and cardiac toxicity are classified as serious adverse events. ${ }^{2}$ Cardiac toxicity of the drug is well known and widely described in the literature, more commonly reported as cases of cardiomyopathy, cardiac rhythm disturbances and conduction impairment..$^{-5}$ Another antimalarial drug is hydroxychloroquine, which also has anti-inflammatory and immunosuppressive effects in systemic lupus erythematosus and rheumatoid arthritis, which are registered as its official indications.

Hydroxychloroquine has cumulative activity, but side effects may occur relatively early. Cardiovascular side effects of chloroquine or hydroxychloroquine like cardiomyopathy, can lead to heart failure, may be fatal in some cases. Conduction disorders are the main side effect reported in patients who receive these drugs. Clinicians should be cautioned that cardiovascular side effects, even conduction abnormalities without severe consequences, associated with the use of chloroquine or hydroxychloroquine, may be initial toxicity manifestations and potentially irreversible..$^{6-8}$ Older patients undergo age-related physiological changes that are manifested in the changes of drug pharmacodynamics, potentially causing increased drug interactions and risk of side effects. Drug interactions and side effects associated with the use of antimalarial drugs in elderly may occur more frequently due to QT prolongation, decreased renal elimination, and decreased hepatic metabolism [9]. Hydroxychloroquine prolongs the QT interval and should not be prescribed in combination with other drugs that have the potential for cardiac arrhythmias. There is an increased risk of ventricular arrhythmias when hydroxychloroquine is used concomitantly with other arrhythmic agents.

The choice of antibacterial agent in favor of azithromycin in the COVID-19 treatment regimens in combination with chloroquine or hydroxychloroquine rises concerns about two aspects: increased risk of cardiac arrhythmic side effects and more frequently reported resistance to monotherapy with this drug, which may affect the pneumonia treatment effectiveness in a patient with COVID-19. In addition, the decrease in treatment effectiveness may be exacerbated by additional immunosuppression and depressed intrinsic immunity associated with the administration of antimalarial drugs such as chloroquine or hydroxychloroquine. Many studies suggest that macrolide therapy was associated with the risk of cardiac complications. ${ }^{10-13}$ Mefloquine is another antimalarial drug developed in the USA in the early 1970 s. ${ }^{14}$ It has no cardiac side effects but has psychoneurological side effects. ${ }^{15}$

A systematic literature review to identify a critical assessment of further use of mefloquine in connection with the risk of psychiatric disorders and suicide risk did not reveal any critical comments supporting these risks in clinical practice for prophylactic use of mefloquine. ${ }^{16}$ Another study conducted on mefloquine, along with cepharanthine and selamectin as a treatment for COVID-19 demonstrated that these drugs showed complete inhibition of cytopathic effects in cell culture. ${ }^{17}$ Given the described drug interactions and possible side effects, clinicians should be vigilant in selecting optimal therapy on a case-by-case basis considering the presence of certain diseases and characteristics of particular patient. It should be remembered that patients with high viral load may develop myocarditis ${ }^{18}$ as a consequence of direct viral toxicity to the myocardium and myocardial damage, as evidenced by studies of troponin level changes and prognosis in this population. ${ }^{19}$ In this case, it should be noted that the prescription of drugs with cardiotoxicity should be limited. To reduce the risk of cardiotoxicity-related side effects, prior to drug prescription, a patient should be evaluated for the following conditions that contraindicate hydroxychloroquine like severe cardiac abnormalities, including significant rhythm and conduction abnormalities, chronic heart failure, cardiomyopathy, marked left ventricular hypertrophy, planned amiodarone or carbamazepine administration. The combination of azithromycin with mefloquine, rather than chloroquine/hydroxychloroquine, appears to be more grounded in terms of safety.

However, patients with previously reported ventricular tachycardia and/or significant QT prolongation should probably refrain from azithromycin. To control cardiotoxicity and safety of the conducted therapy, instrumental and clinical monitoring, including ECG monitoring is required prior to treatment and over time, in patients at high risk, and in patients with a history of cardiovascular diseases regardless of their age. The discussed antiviral drug therapy could be considered as a pathogenetically justified approach in the treatment of the novel coronaviral infection, but an effective antiviral drug with a clearly proven effect on COVID19 has not yet been determined. ${ }^{20}$ Antiviral drugs, remdesivir and favipiravir, currently being actively 
studied in clinical trials in patients with COVID-19, are candidates for the potential use in clinical practice. In addition, intravenous immunoglobulin and donor convalescent plasma donated through plasmapheresis by the patients, who have had the infection, within 2 weeks of recovery and discharge, should be considered another pathogenetically justified strategy for the treatment of COVID-19. Despite all the struggles and hard work behind the research work focused on finding a gold standard treatment for COVID-19, there is no definite and specific outcome.

\section{CONCLUSION}

More in depth understanding of the mode of action of existing drugs against coronavirus with a focus on adverse events caused by them is required. Data on these drugs is very limited in context of COVID-19. Long term trials with large number of subjects need to be conducted to establish and document benefits of these drug combinations so that a significantly substantial evidence can be generated.

\section{REFERENCES}

1. Grasselli G, Zangrillo A, Zanella A, Antonelli M, Cabrini L, Castelli A et al. Baseline Characteristics and Outcomes of 1591 Patients Infected With SARS-CoV-2 Admitted to ICUs of the Lombardy Region, Italy. JAMA. $2020 \quad 28 ; 323(16): 1574-81$. https://doi.org/10.1001/jama.2020.5394.

2. Plantone D, Koudriavtseva T. Current and Future Use of Chloroquine and Hydroxychloroquine in Infectious, Immune, Neoplastic, and Neurological Diseases: A Mini-Review. Clin Drug Investig. 2018;38(8):653-71. https://doi.org/10.1007/s40261-018o656-y.

3. Haeusler IL, Chan XHS, Guérin PJ, White NJ. The arrhythmogenic cardiotoxicity of the quinoline and structurally related antimalarial drugs: a systematic review. BMC Med. 2018;16(1):200. https://doi.org/10.1186/s12916-018-1188-2.

4. Blignaut M, Espach Y, van Vuuren M, Dhanabalan $\mathrm{K}$, Huisamen B. Revisiting the Cardiotoxic Effect of Chloroquine. Cardiovasc Drugs Ther. 2019 Feb;33(1):111. https://doi.org/10.1007/s10557-018-06847-9.

5. Yogasundaram H, Hung W, Paterson ID, Sergi C, Oudit GY. Chloroquine-induced cardiomyopathy: a reversible cause of heart failure. ESC Heart Fail. 2018 Jun;5(3):372-5. https://doi.org/10.1002/ehf2.12276.

6. Chatre C, Roubille F, Vernhet H, Jorgensen C, Pers YM. Cardiac Complications Attributed to Chloroquine and Hydroxychloroquine: A Systematic Review of the
Literature. Drug Saf. 2018;41(10):919-31. https://doi.org/10.1007/s40264-018-0689-4.

7. Dogar MU, Shah NN, Ishtiaq S, Shah PN, Shah P, Mathew $S$ et al. Hydroxychloroquine-induced restrictive cardiomyopathy: a case report. Postgrad Med J. 2018;94(1109):185-6. https://doi.org/10.1136/postgradmedj-2017-135236.

8. Mollerach FB, Scolnik M, Catoggio LJ, Rosa J, Soriano ER. Causes of fetal third-degree atrioventricular block and use of hydroxychloroquine in pregnant women with $\mathrm{Ro} / \mathrm{La}$ antibodies. Clin Rheumatol. 2019;38(8):2211-7. https://doi.org/10.1007/s10067-019-04556-8.

9. Lewis J, Gregorian T, Portillo I, Goad J. Drug interactions with antimalarial medications in older travelers: a clinical guide. J Travel Med. 2020;27(1):tazo89. https://doi.org/10.1093/jtm/tazo89. 10. Polgreen LA, Riedle BN, Cavanaugh JE, Girotra S, London B, Schroeder MC, Polgreen PM. Estimated Cardiac Risk Associated With Macrolides and Fluoroquinolones Decreases Substantially When Adjusting for Patient Characteristics and Comorbidities. J Am Heart Assoc. 2018 21;7(9):eoo8074. https://doi.org/10.1161/JAHA.117.008074.

11. Postma DF, Spitoni C, van Werkhoven $\mathrm{CH}$, van Elden LJR, Oosterheert JJ, Bonten MJM. Cardiac events after macrolides or fluoroquinolones in patients hospitalized for community-acquired pneumonia: post-hoc analysis of a cluster-randomized trial. BMC Infect Dis. 2019 7;19(1):17. https://doi.org/10.1186/s12879-018-3630-7.

12. Trifirò G, de Ridder M, Sultana J, Oteri A, Rijnbeek $\mathrm{P}$, Pecchioli $\mathrm{S}$ et al. Use of azithromycin and risk of ventricular arrhythmia. CMAJ. 2017 18;189(15):E56oE568. https://doi.org/10.1503/cmaj.160355.

13. Yang Z, Prinsen JK, Bersell KR, Shen W, Yermalitskaya L, Sidorova $\mathrm{T}$ et al. Azithromycin Causes a Novel Proarrhythmic Syndrome. Circ Arrhythm Electrophysiol. 2017;10(4):eo0356o. https://doi.org/10.1161/CIRCEP.115.00356o.

14. Honegr K, Dulícek K, Mirovský P, Hozák A. Mefloquine in the treatment of malaria--initial experience in Czechoslovakia. Cesk Epidemiol Mikrobiol Imunol. 1987;36(5):292-6.

15. Jensen JJ. Mefloquine: neuropsychiatric adverse effects are often severe and persistent long after withdrawal of the drug. Ugeskr Laeger. 1998;16o(16):2413.

16. Tickell-Painter M, Saunders R, Maayan N, Lutje V, Mateo-Urdiales A, Garner P. Deaths and parasuicides 
associated with mefloquine chemoprophylaxis: A systematic review. Travel Med Infect Dis. 2017;20:5-14. https://doi.org/10.1016/j.tmaid.2017.10.011.

17. Fan HH, Wang LQ, Liu WL, An XP, Liu ZD, He XQ et al. Repurposing of clinically approved drugs for treatment of coronavirus disease 2019 in a 2019-novel coronavirus-related coronavirus model. Chin Med J (Engl). $\quad 2020 \quad$ 5;133(9):1051-6. https://doi.org/10.1097/CM9.000oooooooooo797. 18. Kim IC, Kim JY, Kim HA, Han S. COVID-19-related myocarditis in a 21-year-old female patient. Eur Heart
J. https://doi.org/10.1093/eurheartj/ehaa288.

19. Guo T, Fan Y, Chen M, Wu X, Zhang L, He T, et al. Cardiovascular Implications of Fatal Outcomes of Patients With Coronavirus Disease 2019 (COVID-19). JAMA Cardiol. $2020 \quad$ 1;5(7):811-8. https://doi.org/10.1001/jamacardio.2020.1017.

20. Sanders JM, Monogue ML, Jodlowski TZ, Cutrell JB. Pharmacologic Treatments for Coronavirus Disease 2019 (COVID-19): A Review. JAMA. 2020 12;323(18):1824-36.

https://doi.org/10.1001/jama.2020.6019.

Source of support: Nil, Conflict of interest: None declared

Cite this article as:

Khullar V . The Potential Adverse Reactions of Administering Combination Therapy in Covid-19 Patients. Int Healthe Res J. 2020;4(9):RV7-RV10. https://doi.org/10.26440/IHRJ/0409.12376

\section{AUTHOR AFFILIATIONS: $\left({ }^{*}\right.$ Corresponding Author) \\ 1. BDS, Private Practitioner, Hoshiarpur, India}

Address of Corresponding Author: House No. 200

Ward no 7

VPO- Urmar Tanda,

Distt. Hoshiarpur, Punjab 